hep-th/9604107

\title{
A SUPERMEMBRANE DESCRIPTION OF STRING-STRING DUALITY
}

\author{
Fermin ALDABE币 \\ Theoretical Physics Institute, University of Alberta \\ Edmonton, Alberta, Canada, T6G 2J1
}

July 20, 2018

\begin{abstract}
We show that the open membrane action on $T^{3} \times S^{1} / Z^{2}$ is equivalent to the close membrane action on K3. The main difference between the two actions is that one generates the KK modes in the worldvolume action which is the strong coupling limit of type IIA superstring while the other action generates the KK modes in a worldsheet action. Thus explaining membrane-string duality in $\mathrm{D}=7$, which naturally leads to string-string duality in $\mathrm{D}=6$.
\end{abstract}

${ }^{1}$ E-mail: faldabe@phys.ualberta.ca 
The spacetime membrane/string duality in seven dimensions was first presented in [1]. This duality can be rephrased, after identification of the orbifolded membrane with the heterotic string [3, 4], in terms of close/open membrane duality. Our strategy to the worldvolume description of membrane/membrane duality will be to show that the bosonic sector of the open membrane action on $T^{3} \times S^{1} / Z_{2}$ is the same as the bosonic sector of the close membrane action on K3 after both theories are dimensionally reduced. The worldvolumes of the close and open membrane are topologically distinct. However, upon dimensionally reducing each theory, the worldvolumes become close strings and are therefore topologically equivalent. This will insure that both theories have the same bosonic massless spectrum, which provided they have the same spacetime supersymmetry insures that both theories have the same massless spectrum.

The action for the bosonic sector of an anomaly free open membrane is [3]

$$
\begin{aligned}
S= & S_{M}+\int_{\partial M^{3}}\left\{\frac{1}{2}\left(g_{m n} \eta^{i j}+b_{m n} \epsilon^{i j}\right) \partial_{i} x^{m} \partial_{j} x^{n}\right. \\
& \left.+\frac{1}{2}\left(g_{I J} \eta^{i j}+b_{I J} \epsilon^{i j}\right) \partial_{i} x^{I} \partial_{j} x^{I}+\epsilon^{i j} \partial_{i} x^{J} \partial_{j} x^{m} A_{m}^{J}(x)\right\}
\end{aligned}
$$

where

$$
S_{M}=\int_{M^{3}}\left(\sqrt{-g_{m n} \partial_{i} x^{m} \partial_{j} x^{n}}+\frac{1}{6} \epsilon^{i j k} \partial_{i} x^{m} \partial_{j} x^{n} \partial_{k} x^{p} B_{m n p}\right),
$$

$g_{m n}$ is the metric on $M^{11}, x^{m}$ are coordinates on $M^{11}$, and $B_{m n p}$ is an antisymmetric 3-tensor. The worldvolume $M^{3}$ is $R \times S^{1} \times S^{1} / Z_{2}$. The 16 left moving bosons $x^{J}, J=1, \ldots, 8$ live only on the boundary of the open membrane, which is two copies of $R \times S^{1}$. They couple naturally to $A^{J}$, the $U(1)$ connections.

The double dimensional reduction of the twisted supermembrane on $M^{10} \times S^{1} / Z_{2}$ of (11) was given in [3]. The bosonic sector is that of the heterotic string

$$
\begin{aligned}
S_{h}= & \int d^{2} \sigma\left\{\frac{1}{2}\left(g_{m n} \eta^{i j}+b_{m n} \epsilon^{i j}\right) \partial_{i} x^{m} \partial_{j} x^{n}\right. \\
& \left.+\frac{1}{2}\left(g_{I J} \eta^{i j}+b_{I J} \epsilon^{i j}\right) \partial_{i} x^{I} \partial_{j} x^{I}+\epsilon^{i j} \partial_{i} x^{I} \partial_{n} x^{m} A_{m}^{(I)}(x)\right\},
\end{aligned}
$$

and the gauge group indices are now $I=1, \ldots, 16$. 
We now consider the case in which $M^{10}=T^{3} \times M^{7}$ where $\operatorname{dim} H^{1}\left(M^{7}\right)=0$. The worldsheet action is a sum of three terms

$$
\begin{aligned}
S_{\text {het }} & =S_{s t}+S_{K K}+S_{\text {mod }} \\
S_{s t} & =\int d^{2} \sigma \frac{1}{2}\left(g_{m n} \eta^{i j}+b_{m n} \epsilon^{i j}\right) \partial_{i} x^{m} \partial_{j} x^{n} \\
S_{K K} & =\int d^{2} \sigma \epsilon^{i j} \partial_{i} x^{I} \partial_{j} x^{m} A_{m}^{I} \\
S_{\text {mod }} & =\int d^{2} \sigma \frac{1}{2}\left(g_{I J} \eta^{i j}+b_{I J} \epsilon^{i j}\right) \partial_{i} x^{J} \partial_{j} x^{I}
\end{aligned}
$$

The index $I=1, \ldots, 22$ labels 22 gauge fields: 16 come from the internal dimensions of the heterotic string, and the other 6 gauge fields are the KK modes of the metric and antisymmetric tensor. Sixteen of the $x^{I}$ 's are left moving bosons, the remaining ones are both left-right moving bosons. The action $S_{\text {mod }}$ has a massless spectrum given by moduli fields which correspond to deformations of the Narain lattice and therefore take values on the group manifold

$$
\frac{S O(19,3)}{S O(19) \times S O(3)} \text {. }
$$

Something is very peculiar to action (4). All the gauge fields have appeared within a two-dimensional theory, and not a three-dimensional theory. This is exactly what happens in the long wavelength limit of the open membrane: the gauge fields are defined in terms of fields which live on 10-dimensional boundaries of M-theory. This does not happen in the long wavelength limit of the close membrane: there, the gauge fields are defined in terms of 11-dimensional fields. We should then expect the gauge fields of the close membrane to be defined over $M^{3}$ and not over its boundary which is absent for the close membrane.

The closed membrane action on $K 3 \times M^{7}$ is

$$
S_{M}^{\prime}=\int_{M^{3}} d^{3} \zeta\left(\sqrt{-g_{m n} \partial_{i} x^{m} \partial_{j} x^{n}}+\frac{1}{6} \epsilon^{i j k} \partial_{i} x^{m} \partial_{j} x^{n} \partial_{k} x^{p} B_{m n p}\right) .
$$

where $M^{3}$ is $T^{2} \times R$, and spacetime is $M^{7} \times K 3$. In terms of compact indices, $a, b, \ldots$ and spacetime indices, $m, n, \ldots$, action (6) becomes

$$
S_{M}^{\prime}=S_{s t}^{\prime}+S_{K K}^{\prime}+S_{m o d}^{\prime}
$$




$$
\begin{aligned}
S_{s t}^{\prime} & =\int d^{3} \sigma \sqrt{-g_{m n} \partial_{i} x^{m} \partial_{j} x^{n}}+\frac{1}{6} B_{m n p} \epsilon^{i j k} \partial_{i} x^{m} \partial_{j} x^{n} \partial_{k} x^{p} \\
S_{K K}^{\prime} & =\frac{1}{6} \int d^{3} \sigma \epsilon^{i j k} \partial_{i} x^{a} \partial_{j} x^{b} \partial_{k} x^{m} B_{a b m} \\
S_{m o d}^{\prime} & =\int d^{3} \sigma \sqrt{-g_{a b} \partial_{i} x^{a} \partial_{j} x^{b}}+\frac{1}{6} \epsilon^{i j k} \partial_{i} x^{a} \partial_{j} x^{b} \partial_{k} x^{c} B_{a b c}
\end{aligned}
$$

The term $S_{K K}^{\prime}$, has only one relevant term because K3 surfaces have no onecycles. The three-form potential in $S_{K K}^{\prime}$, in analogy with the two-form potential of the heterotic string, generates KK modes. The three-form potential that appears in $S_{K K}^{\prime}$ of action (7) can be expanded in terms of the cocycles of K3. For the 22 two-cocycles of K3, we may decompose $B$ in an analogous manner as used in [6] for the two-form potential

$$
B_{a b m}=b_{a b}^{I}\left(x^{a}\right) C_{m}^{I}\left(x^{r}\right)
$$

where $I=1, \ldots, 22$ labels the two-cycles of $K 3$. Inserting (8) into $S_{K K}^{\prime}$ yields

$$
\int_{M^{3}} \epsilon^{i j k} \partial_{i} x^{m} \partial_{j} x^{b} \partial_{k} x^{a} b_{a b}^{I}\left(x^{c}\right) C_{m}^{I}\left(x^{r}\right) .
$$

We now use reparametrization invariance to set

$$
\rho=x^{11},
$$

where $\rho$ is a worldvolume coordinate, and perform a dimensional reduction of (9). However, to do so, we first review some facts about membrane/string duality of the low energy theory in $\mathrm{D}=7$ [1, 2].

The kinetic terms for the gauge fields in $D=7$ supergravity read

$$
\int_{M^{7}} \sqrt{-g^{(7)}} a_{I J} F_{m n}^{I} F^{J m n}
$$

and can be obtained after a split of the four-form field strength, $H=d B$, of the 11-dimensional supergravity action

$$
H_{a b m n}=b_{a b}^{I} F_{m n}^{I},
$$


from the term

$$
\int_{M^{11}} \sqrt{-g^{(11)}} H^{2}=\int_{M^{7}} \sqrt{-g^{(7)}} F_{m n}^{I} F^{J m n} \int_{K 3} \sqrt{-g^{(K 3)}} b_{a b}^{I} b^{J a b} .
$$

Membrane/string duality in $\mathrm{D}=7$ requires the existence of a point in the moduli space of K3 where all the 22 gauge fields are enhanced[2]. An enhancement of a U(1) gauge field occurs, as argued in [2], when a 2-cycle vanishes and two solitonic membranes charged with respect to a $\mathrm{U}(1)$ gauge field become massless. What this means is that even though a 2-cycle vanishes, the 2-form dual to the vanishing 2-cycle is still well defined. Otherwise, we see from (13), that the U(1) symmetry would not be present at the point in the moduli space since some $b_{a b}^{I}$ would not be defined. Therefore, in enhancing simultaneously the 22 gauge symmetries we must require the 222 -cycles of K3 to vanish and the existence of a limiting procedure in which the 22 elements, $b^{I}$, of $H^{2}(K 3)$ are well defined at that point in the moduli where such an enhancement of symmetry takes place. Otherwise membrane/string duality would not hold. The 22 two-cocycles, $b^{J}$ are defined only over their dual two-cycles, $\gamma_{J}$, through the relation

$$
\int_{\gamma_{I}} b^{J}=\delta_{I J}
$$

Then, we expect that the $22 b^{I}$ 's will be coordinate independent when the $22 \gamma_{I}$ 's vanish to a point. Therefore, at the point in the moduli space when the 22 two-cycles vanish it holds that

$$
\begin{aligned}
& \partial_{x^{11}} b_{a b}^{I}=0 \\
& \partial_{x^{11}} g_{a b}^{I}=0
\end{aligned}
$$

since the metric, $g_{a b}$, on $\mathrm{K} 3$ is also defined in terms of elments of $H^{2}(K 3)$. At the point in the moduli space where (15) holds, we can also require that

$$
\partial_{\rho} x^{M}=0 \quad M \neq 11 .
$$

\footnotetext{
${ }^{2}$ This also occurs at a point in the moduli space of the heterotic string on $T^{3}$.
} 
This conditions are sufficient to perform a dimensional reduction which yields

$$
\int_{M^{2}} \epsilon^{i j} \partial_{i} x^{m} \partial_{j} x^{b} b_{11 b}^{I} C_{m}^{I}
$$

Thus, identifying $C^{I}$ with $A^{I}$ and $\partial_{j} x^{b} b_{11 b}^{I}$ with $\partial_{j} x^{I}$, we are able to match the gauge sector of the close membrane with the gauge sector of the open membrane. This means that the map

$$
\begin{aligned}
b_{a 11}^{I} \partial_{j} x^{a} & \rightarrow \partial_{j} x^{I} \\
C_{m}^{I} & \rightarrow A_{m}^{I}
\end{aligned}
$$

which we will refer to as S-duality map, takes action (17) to the form

$$
\int_{M^{2}} \epsilon^{i j} \partial_{i} x^{I} A_{m}^{I}
$$

which is equivalent to the term $S_{K K}$ in (4). The map (18) does not act on $x^{11}$. This map acts on the induced metric on the worldvolume. Thus, the term in $S_{\text {mod }}^{\prime}$ in (7) yields after a double dimensional reduction of $x^{11}$

$$
\int d^{2} \sigma \frac{1}{2}\left(g_{I J} \eta^{i j}+b_{I J} \epsilon^{i j}\right) \partial_{i} x^{J} \partial_{j} x^{I}
$$

where

$$
\begin{aligned}
& g_{I J}=g_{a b} b_{I}^{11 a} b_{J}^{11 b}, \\
& b_{I J}=B_{a b 11} b_{I}^{11 a} b_{J}^{11 b} .
\end{aligned}
$$

Action (20) is equivalent to $S_{m o d}$ in eq. (4). Thus, the S-duality map which takes $S_{K K}$ to $S_{K K}^{\prime}$ also takes $S_{m o d}$ to dimensionally reduced $S_{m o d}^{\prime}$.

In order to arrive to this matching of the gauge sectors of the close and open membrane, it is necessary to generate the gauge fields of the closed membrane before dimensionally reducing the theory, as opposed to the gauge fields of the open membrane which are always generated within the two-dimensional theory. This explains the origin of strong-weak duality in string theory. The strong coupling limit of of type IIA string is 11-dimensional supergravity which is believed to arise as the long 
wavelength limit of supermembrane theory. Thus, gauge fields present in the three dimensional theory will be strongly interacting, and will continue to be strongly interacting after dimensional reduction to a two-dimensional theory. On the other hand, the open membrane has its gauge fields appearing in two dimensional theories, which are therefore weakly interacting.

We must now consider the spacetime part of the action for the close membrane (2). The term

$$
\int_{M^{3}} \sqrt{-g_{m n} \partial_{i} x^{m} \partial_{j} x^{n}}
$$

where $i j$ label coordinates on $M^{3}$, is independent of $x^{11}$ and therefore independent of $\rho$. It can be dimensionally reduced using the procedure of [5] which yields

$$
\int_{M^{2}} \sqrt{-g_{m n} \partial_{i} x^{m} \partial_{j} x^{n}}
$$

where $i j$ now label coordinates on $M^{2}$. This term coincides with the first term in $S_{s t}$ in (4).

The term

$$
\int_{M^{3}} \epsilon^{i j k} \partial_{i} x^{m} \partial_{j} x^{n} \partial_{k} x^{p} B_{p n m}
$$

can be mapped to a term [6]

$$
\int_{W} d \Sigma^{m n p q} H_{m n p q}
$$

where $H=d B$ and $W$ is an element of $H_{4}\left(M^{7}\right)$. This term is topological, and duality of the seven dimensional space means that $H^{3}\left(M^{7}\right)=H^{4}\left(M^{7}\right)$. Therefore (25) can be written as

$$
\int_{* W} d \Sigma^{m n p} H_{m n p}
$$

where $* W$ is the Hodge dual of $W$. which again can be mapped to a term [6]

$$
\int_{M^{2}} \epsilon^{i j} \partial_{i} x^{m} \partial_{j} x^{n} b_{n m}
$$


Thus, the b-term in the space time string action is a direct consequence of the duality of the seven dimensional duality between 3- and 4-forms, just as it is the case for the low energy actions [1]. We then learn that the term (27) is equivalent to the second term in $S_{s t}$ in (弣).

Thus, the dimensional reduction of $S_{s t}^{\prime}$ in (7) yields the term $S_{s t}$ in (4), and therefore, we have succeeded in mapping the close membrane action on K3 to the open membrane action on $T^{3} \times S^{1} / Z^{2}$. This shows that as far as the bosonic massless spectrum is concerned, the open and close membrane actions are dual to each other when one is compactified on $T^{3} \times S^{1} / Z_{2}$ and the other on K3. Their bosonic massless spectra, as we have shown are the same because of the duality of seven dimensional spaces, and because the 3-form potential of the close membrane yields the same number of gauge fields and the same moduli as the open membrane compactified on $T^{3} \times S^{1} / Z_{2}$. It remains to show that both theories have the same massless spectra. This will be the case because both theories have the same spacetime supersymmetry

[1]. That is, the S-duality map must also map the fermions which are sections of the tangent bundle of one theory to fermions which are sections of a different tangent bundle, but which preserve spacetime supersymmetry.

\section{Acknowledgements}

I have benefited from discussions with B. Campbell and J.D. Lewis. 


\section{References}

[1] P.K.Townsend, String-Membrane Duality in Seven Dimensions, Phys. Lett. B354 (1995) 247.

[2] C.M.Hull and P.K.Townsend, Enhanced Gauge Symmetries in Superstring Theories, Nucl. Phys. B451 (1995) 525.

[3] F. Aldabe, Heterotic and Type I Strings from Twisted Supermembranes, hepth 9603183.

[4] P. Hořava and E. Witten, Heterotic and Type I String Dynamics From Eleven Dimensions, hepth 9510209;

Eleven-Dimensional Supergravity on A Manifold with Boundary, hepth 9603142.

[5] M.J.Duff, P.S.Howe, T.Inami, and K.S.Stelle, Superstring in D=10 from supermembranes in D=11, Phys. Lett. B191 (1987) 70.

[6] R. Rohm and E. Witten, The Antisymmetric Tensor Field in Superstring Theory, Ann. Phys. 170 (1986) 454. 